\title{
The Role of Biomaterials on Cancer Stem Cell Enrichment and Behavior
}

\author{
FARIDE ORDIKHANI, ${ }^{1}$ YONGHYUN KIM ${ }^{2,3}$ and SILVIYA P. ZUSTIAK ${ }^{1,4}$ \\ 1.-Department of Biomedical Engineering, Saint Louis University, 3507 Lindell Blvd, St Louis, \\ MO, USA. 2.-Department of Chemical and Biological Engineering, The University of Alabama, \\ Box 870203, Tuscaloosa, AL, USA. 3.—e-mail: ykim@eng.ua.edu. 4.—e-mail: szustiak@slu.edu
}

\begin{abstract}
The theory of cancer stem cells (CSCs) and their role in cancer metastasis, tumorigenicity and resistance to therapy is slowly shifting the emphasis on the search for cancer cure: more evidence is surfacing that a successful therapy should be geared against this rare cancer cell population. Unfortunately, CSCs are difficult to culture in vitro which severely limits the progress of CSC research. This review gives a brief overview of CSCs and their microenvironment, with particular focus on studies that used in vitro biomaterial-based models and biomaterial/CSC interfaces for the enrichment of CSCs. Biomaterial properties relevant to CSC behaviors are also addressed. While the discussed research field is still in its infancy, it appears that in vitro cancer models that include a biomaterial can support CSC enrichment and this has proved indispensable to the study of their biology as well as the development of novel cancer therapies.
\end{abstract}

\section{INTRODUCTION}

The hypothesis of cancer stem cells (CSCs) is becoming increasingly prominent in cancer research due to the increased recognition of the link between cancer cell pluripotency and increased metastasis and irresponsiveness to treatments. CSCs are a specialized subset of tumor cells with stem cell-like properties, such as self-renewal and differentiation potential (collectively, "stemness"). ${ }^{1-3}$ CSCs are hypothesized to be at the apex of the tumor cell hierarchy and are the only subset of cells capable of initiating tumors that comprise CSCs and non-stem bulk tumor cells. The exact origin of CSCs is unclear. ${ }^{4}$ It is currently being debated whether they arise from malignantly transformed stem cells or from dedifferentiated mature cells, which is why some prefer to name them instead as tumor-initiating cells (TICs). ${ }^{5}$ Regardless of their origin or name, CSCs/TICs have been demonstrated to exist in various solid tumors, including breast, brain, and colon tumors. ${ }^{6-9}$ Functionally, they have also been shown to have heightened chemo- and radio-resistance and thus explain the tumor relapse observed in many patients. ${ }^{1,2}$ Because of these characteristics, CSCs are postulated to promote the epithelialto-mesenchymal transition (EMT) during cancer metastasis. ${ }^{10-12}$ CSCs are thus positioned as the main barrier against cancer cures but also a hope in the search for them. ${ }^{13}$

Unfortunately, CSCs are difficult to propagate in vitro due to the difficulty of recapitulating the necessary CSC niche, ${ }^{14}$ which severely limits the study of CSC biology as well as therapy development. ${ }^{15}$ Typically, CSCs are propagated and studied in vivo or in tumor spheroid models grown on non-adhesive substrates and serum-free media. ${ }^{16}$ Here, we suggest that improved cancer models will further support CSC enrichment and that such in vitro models will include clever biomaterial designs. ${ }^{17}$ Biomaterials, and more specifically hydrogels with their extracellular matrix (ECM)like properties, hydrophilicity and biocompatibility have become essential components in the study of mammalian cells, including stem cells. ${ }^{18}$ By careful material design and selection of appropriate mechanical, physical and biochemical properties, we are now able to expand stem cells or direct their fate. ${ }^{18}$ For example, biomaterials afford the ability to fine tune the matrix stiffness that better mimics the stem cell niche. ${ }^{19-21}$ These studies show that stiffness and mechanical matching could direct the fate of stem cells, instructing the stem cells to produce differentiated progeny cells rather than 
self-renew. Insights into the striking similarities between stem cells and CSCs have surfaced; ${ }^{1}$ thus, one is left to wonder if hydrogel biomaterials or biomaterials in general would have the same impact on CSC research. While we do not currently have sufficient information to form definitive conclusions on the role of biomaterials on CSC fate, recent studies suggest that biomaterials might be the key to enriching the rare CSC populations and gaining a deeper understanding of their role in cancer pathology and resistance to therapy.

\section{CANCER STEM CELLS (CSCS) AND THEIR MICROENVIRONMENT}

Much of our current understanding of CSCs is derived from various animal studies. ${ }^{6-9,22}$ For example, to test for tumor initiation, serial limiting dilutions of tumor cells are orthotopically xenografted into a cohort of immunocompromised mice and tumor formation in the animals is measured. The cell population that is capable of forming tumors even at very dilute concentrations are considered to be enriched for CSCs. Candidate therapeutics are then tested in animal models to demonstrate their efficacy and to predict success in the clinic. While these preclinical animal models are still considered the gold standard for CSC identification and functional assays, they are very laborious and expensive to generate. In many cases, animal models also fail to accurately predict clinical outcomes. ${ }^{23}$ The results are often convoluted with complex sets of variables, such as animal-to-animal variations and the role of an intact immune system which is present in human patients but not properly modeled in most xenograft models. These and many other factors often lead to poor translation from preclinical to clinical studies. Another common problem with these systems is the lack of appropriate control that in vitro systems can afford. There is therefore a need to create simple, yet robust in vitro systems that can better recapitulate and model the CSC biology.

CSCs have been demonstrated to be present in various solid tumors, including the breast, brain, and colon tumors. ${ }^{6-9}$ In each of these solid tumors, the CSC subpopulation has been identified to have unique specific set of markers. Breast CSCs are identified as CD $44^{+} / \mathrm{CD} 24^{-}$cells, ${ }^{6}$ whereas $\mathrm{CD} 133^{+}$ marks the CSCs of the colon ${ }^{8,9}$ and brain tumors. ${ }^{7}$ While CD44 can be activated by its substrate hyaluronic acid, ${ }^{11}$ the exact functional significance of these CSC markers remain unknown. ${ }^{24-26}$ The in vitro microenvironment was found to have an impact on CSCs and their expression of these markers. CSCs are often lost in established cancer cell line cultures that are grown in serum, and these results have necessitated the use of primary cells in defined serum-free media instead. ${ }^{27,28}$ Hence, there is a clear need to better understand CSC biology and develop methods to model and screen therapeutics against them.
It is well established that the microenvironment of tumor cells plays an important role in their biology. ${ }^{29}$ Certain microenvironment (such as hypoxia, acidic $\mathrm{pH}$, low nutrient, and presence of stromal cells) can help increase the aggressiveness of the tumor cells and cause them to acquire invasive characteristics. For example, hypoxic conditions may lead tumor cells to secrete angiogenic factors (e.g., vascular endothelial growth factor, VEGF) to recruit or initiate new blood vessel formation (i.e., tumor-induced angiogenesis). ${ }^{30,31}$ Acidic $\mathrm{pH}$ has also been found to promote an increase of cancer stem cell phenotype. ${ }^{32}$ Furthermore, nutrient stress has also been implicated in enhancing cancer stem cell phenotype through Wnt signaling, ${ }^{33,34}$ and stromal cells were found to mediate cancer cell adhesion and motility. ${ }^{35-37}$ Thus, there is a bi-directional effect of the tumor microenvironment in which the environment affects the tumor cell characteristics and in return the tumor cells remodel their microenvironment. In particular, it is believed that CSCs are especially efficient in modulating their microenvironment. ${ }^{8,39}$

One specific way that CSCs may be involved in interacting with their microenvironment is during metastasis. ${ }^{40}$ It has been suggested that they are particularly endowed with the capability to utilize the EMT mechanism. EMT markers such as vimentin and $\mathrm{N}$-cadherin have been observed to be highly expressed in CSCs. ${ }^{41}$ Recently, it was also suggested that CSCs are capable of the reverse process, i.e. mesenchymal-to-epithelial transition (MET). ${ }^{42,43}$ Accordingly, it is becoming apparent that CSCs have robust means to both initiate metastasis and colonize a new distal location for secondary tumor formation. ${ }^{39}$ In all these steps, their sensing and their interplay with the microenvironment is indispensable and elucidating these interactions will become key in developing better in vitro CSC models.

Towards this end, the past several years of development in the bioengineering and tissue engineering fields can offer elegant solutions. Ideally, a model of in vitro tumor environment should be conducible for facile manipulation by researchers, while simultaneously being permissive for CSCs to interact with it and even remodel it. Biocompatible and biodegradable hydrogels offer such highly tunable and employable models.

\section{BIOMATERIALS FOR CSC GROWTH}

Biomaterial-based three-dimensional (3D) culture models that provide direct interactions between cells and between cells and their microenvironment can lead to an increased stemness of cultured cells. They can help promote properties such as cell self-renewal, migration, release of endocrine and paracrine factors that regulate tumor development and angiogenesis, form hypoxic niches, and increase therapy resistance. ${ }^{44}$ Accumulating evidence also indicates that 
(a)



(b)

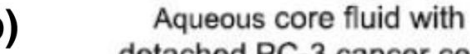

Aqueous core fluid with
detached PC-3 cancer cells

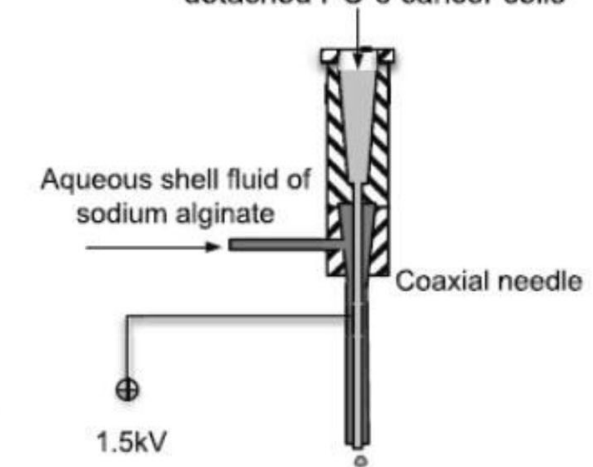

Cancer stem-like cells (CSCs)

- Non-stem cancer cells

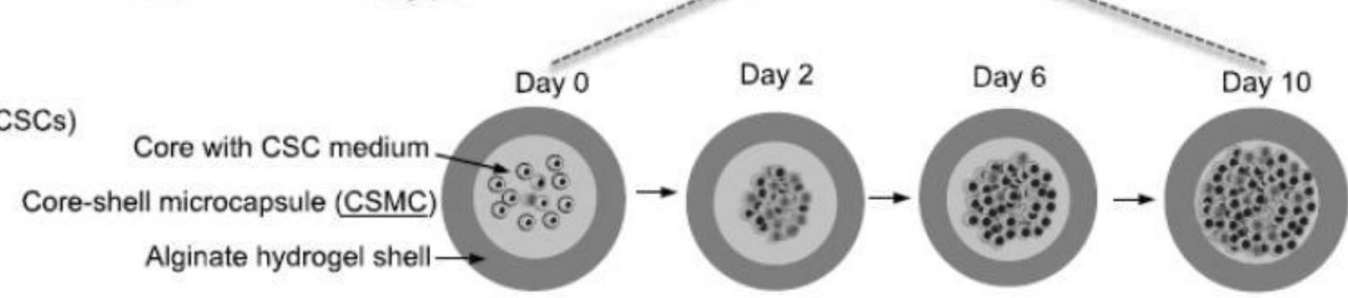

Fig. 1. A schematic of conventional culture in open bulk cancer stem cell (CSC) medium in ultralow attachment plate (ULAP) versus a miniaturized 3D culture in CSC medium in the core of core-shell alginate microcapsules (CSMCs). (a) Conventional culture in ULAP. (b) 3D culture in CSMCs: the cell-laden CSMCs were produced by coaxial electrospray using coaxial needle in one step and transferred into regular 6-well plates for culture in CSC medium. The percentage of CSCs enriched in the spheres is time-dependent and is the highest for spheres obtained from CSMC culture for 2 days. Reprinted with permission from Ref. 17.

cancer cells in $3 \mathrm{D}$ culture display higher malignancy and invasive capability compared to their counterparts in two-dimensional (2D) culture, which has been linked to increased cancer cell stemness. ${ }^{45}$ Consequently, a number of polymers have been utilized to develop 3D cell culture scaffolds for the investigation of proliferation, growth and migration of CSCs.

In a recent study, Rao et al. ${ }^{17}$ reported that encapsulating and culturing PC-3 human prostate cancer cells in miniaturized $3 \mathrm{D}$ alginate hydrogels significantly shortened the time required for obtaining the CSC-containing tumor spheroids (Fig. 1). Moreover, the tumor spheroids obtained on day 2 using the new approach were found to have higher expression of stem cell surface receptor markers, and exhibited higher pluripotency than the ones obtained from conventional non-biomaterial spheroid models. These properties rendered the cells able to possess higher tumorigenicity in vivo. ${ }^{17}$ Interestingly, the biomaterial-grown spheroids also contained more side population cells, a rare CSC subpopulation with increased expression of the adenosine triphosphate (ATP)-binding cassette family of p-glycoproteins (also called multidrug resistance protein 1) that can pump out small molecule drugs from the cells essentially making them drug resistant. ${ }^{17}$ Such direct comparisons of the role of biomaterials over current strategies are still rare in the literature, yet there is a small but growing research niche exploring the possibility of enriching CSCs as well as guiding their behavior with the help of hydrogels, both natural and synthetic.

Hydrogels made of natural materials are generally advantageous because of their intrinsic biocompatibility, biodegradability, and, in many cases, favorable biochemical properties. ${ }^{46}$ Many naturallyoccurring polymers such as alginate (derived from red algae) and chitosan (derived from crustaceans) lack cell-attachment sites and these have to be added independently. ${ }^{47}$ However, inert biomaterials can be used as-is since cell attachment is not necessary for CSC survival and proliferation. ${ }^{1}$ Indeed, 

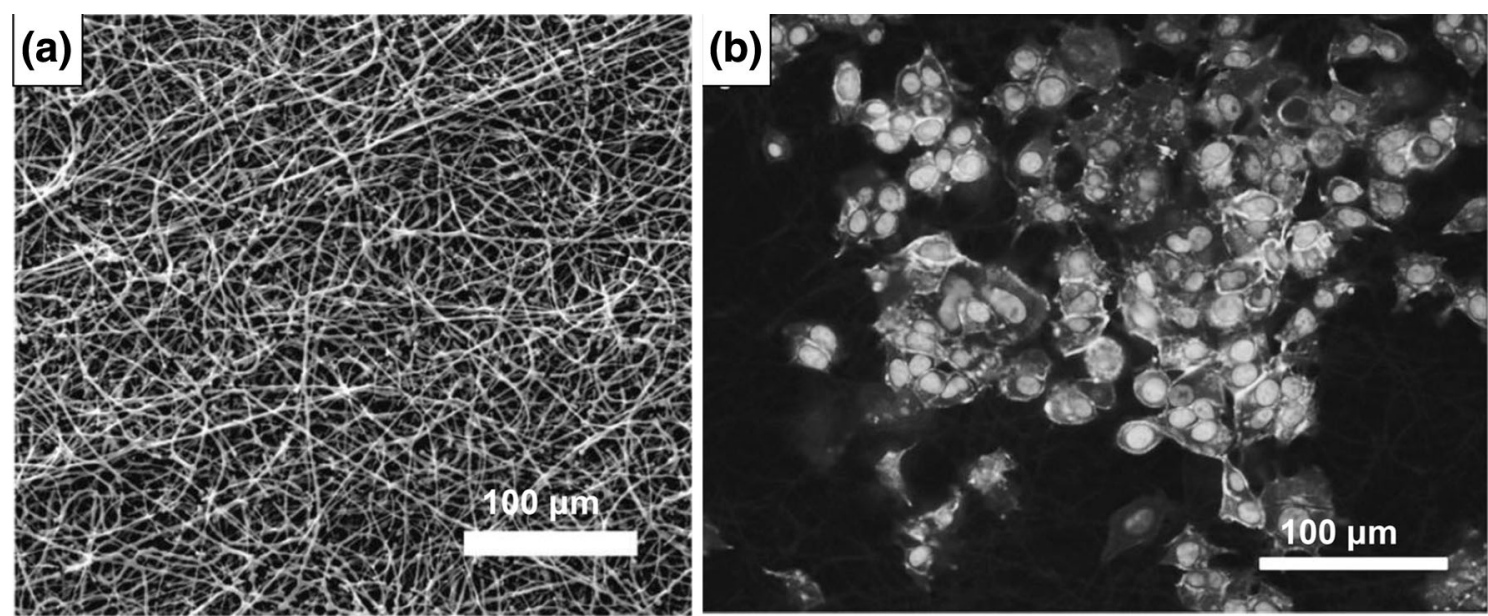

Fig. 2. (a) Scanning electron microscopy image of electrospun polycaprolactone fibrous scaffold. (b) Microscopy image of MCF-7 breast cancer cells cultured in 3D polycaprolactone fibrous scaffold. Reprinted with permission from Ref. 59.

it appears that, even in the absence of receptor ligand-mediated cell-matrix communications, biomaterials can be advantageous. For example, in a recent work, $\mathrm{Xu}$ and coworkers ${ }^{48}$ reported that the proportion of liver cancer cells with CSC-like phenotype as well as cells expressing CSC-related genes was significantly increased in alginate beads compared to 2D culture. Moreover, increased metastasis ability and higher anti-cancer drug resistance were also observed in 3D-cultured cells. ${ }^{48}$ In another study, porous 3D chitosan-alginate scaffolds have been used for the culture of glioblastoma cells showing that the scaffold-grown cells exhibit a more malignant phenotype as well as higher tumorigenicity and angiogenic capacity than cells cultured on $2 \mathrm{D} .{ }^{49}$ In a follow-up study, the authors demonstrated that chitosan-alginate scaffolds promoted the growth and enrichment of the CD $133^{+}$CSC population in glioblastoma cells and that the microenvironment provided by the scaffolds contributed to the more stem-like state of the cells through CD44 (a CSC marker) signaling and EMT. ${ }^{15}$ Lastly, chitosan-alginate scaffolds have also been used to culture liver cancer cell, namely hepatocellular carcinoma (HCC), with similar results: HCC cells grown in 3D scaffolds featured greater angiogenic potential, increased expression of markers for malignancy and migration, rapid transfer into animals, and an increased resistance to chemotherapy in comparison to $2 \mathrm{D}$ cultures. ${ }^{50}$

Another natural material being explored as a scaffold for cancer cells and CSC enrichment is hyaluronan (HA). HA is a natural, anionic, abundant ECM component, which is particularly intriguing due to its role in CD44 binding and its ability to facilitate tumor progression, EMT phenotype, and drug resistance, as well as cell migration and invasion. ${ }^{51,52}$ However, due to its anionic character, HA by itself does not support cell attachment or high cell viability. This drawback could be alleviated by reacting with a second biocompatible polymer such as the cationic chitosan or incorporating $\mathrm{HA}$ as a component in synthetic scaffolds. For example, when incorporated in a 3D polycaprolactone scaffold, HA has been demonstrated to induce a more undifferentiated cell phenotype of glioma cells, i.e. to promote CD133 overexpression, making it useful for the study of glioma CSCs. ${ }^{53}$ Porous HA-chitosan scaffolds have also been used to culture multicellular spheroids composed of glioblastoma ${ }^{54}$ and non-small cell lung cancer cells, ${ }^{55}$ noting increased spheroid formation, stem-like properties, and invasiveness in both cases. Additionally, non-small cell lung cancer cells revealed more EMT markers as well as exhibited 5-6 times higher resistance to cisplatin and 16-56 times higher resistance to methotrexate compared to conventional $2 \mathrm{D}$ cultures. ${ }^{55}$

A common feature of the natural hydrogels discussed above is that they lack cell attachment sites. Unlike them, collagen, the main constituent of the ECM of most tissue types, is capable of providing cell attachment sites and thus activate integrin binding signaling pathways. ${ }^{56}$ Not surprisingly, collagen has also been studied for its ability to enrich the CSC population. Chen et al. observed that a 3D collagen scaffold promoted overexpression of pro-angiogenic growth factors, enhanced the tumorigenicity of breast cancer cells in vivo, upregulated EMT markers, and enriched the CSC population. ${ }^{45}$ Adenoid cystic carcinoma cells have also been cultured in collagen matrices with similar effects of increased angiogenesis and up-regulation of CSC and EMT markers. ${ }^{57}$ In addition, the authors observed increased resistance to chemotherapeutic drugs when the cells were cultured in the $3 \mathrm{D}$ matrix. ${ }^{57}$

A few synthetic scaffolds have also been used to produce 3D matrices for the study of CSC biology and pathology. An added benefit of synthetic 


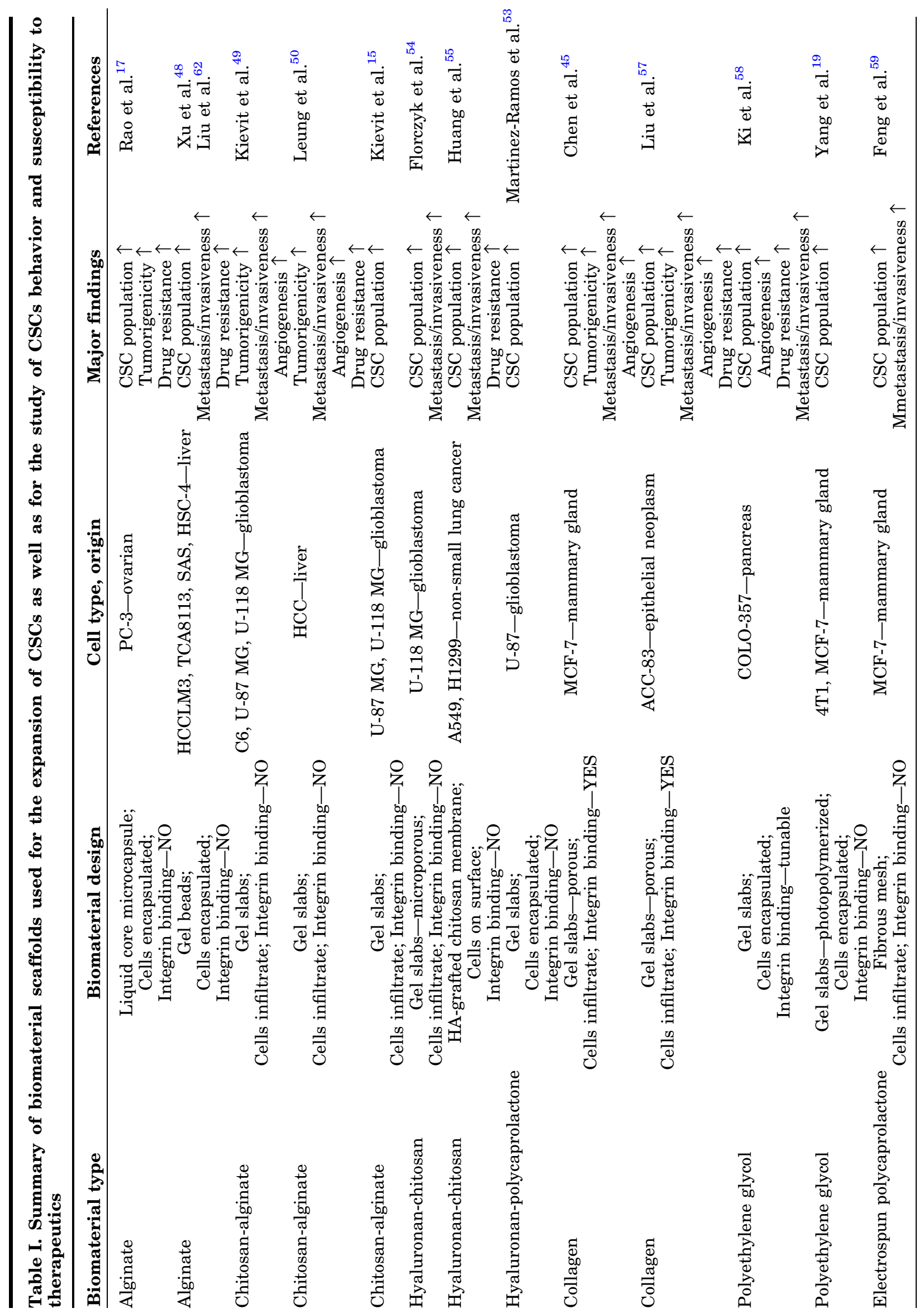


hydrogels over natural materials is the improved flexibility to design $3 \mathrm{D}$ matrices with a wide range of mechanical, physical, and biological properties that are crucial to the maintenance, differentiation, maturation, and fate of the encapsulated cells. ${ }^{19}$ For example, an enzymatically degradable polyethylene glycol hydrogels with orthogonally controlled biophysical and biochemical properties have been developed for the study of pancreatic ductal adenocarcinoma. ${ }^{58}$ When collagen was added to the matrix (i.e. in the presence of cell attachment), there was a greater cell spreading and an up-regulation of membrane type 1-matrix metalloproteinase 1 (MT1-MMP; i.e. enzymes for matrix degradation), suggestive of EMT phenotype. ${ }^{58}$ Interestingly, irrespective of collagen presence, cells cultured in 3D matrices developed chemo-resistance, likely as a consequence of the enrichment of pancreatic CSCs that expressed high levels of CD24, sonic hedgehog (a protein that controls cell division of adult stem cells and is implicated in the development of some cancers), and VEGF. ${ }^{58}$ Lastly, fibrous electrospun polycaprolactone scaffolds have been tested for the expansion of breast cancer cells and showed an increased proportion of CSCs. ${ }^{59}$ Moreover, an upregulation of EMT and increased invasive capability were observed in cells cultured on fibrous scaffolds. ${ }^{59}$ Note that in this case, unlike the hydrogel biomaterial scaffolds discussed above, the electrospun scaffold presented a very different architecture to the cells-randomly oriented fibers devoid of cell attachment sites (Fig. 2) - yet had similar outcomes. ${ }^{59}$

To summarize, it appears that integrin binding is not necessary to promote enrichment of CSC populations. Furthermore, integrin binding does not seem to affect invasiveness, tumorigenicity, angiogenesis or, in some cases, drug resistance in 3D matrices, irrespective of the material or cell type. On the other hand, encapsulation in a 3D matrix, irrespective of the matrix structure (i.e. nano- or microporous, or fibrous), seems to enhance all of the above cell behaviors. This generalization does not imply that matrix dimensionality is important while integrin signaling is not; rather, it is an avenue that needs further exploration. In addition, the modulus of the material is another key property that affects cell migration, proliferation, and spreading, as well as stem cell propagation and differentiation. ${ }^{60,61}$ Recent studies suggest that material stiffness can also affect CSCs and that the trend is not linear. For example, Yang et al. used an inert polyethylene glycol with a range of modulus of $2.5-70 \mathrm{kPa}$ to study the effect of stiffness on CSC marker expression and tumor spheroid formation. ${ }^{19}$ Results showed that the gel modulus had a strong bimodal effect on the tumor spheroid formation and the expression of CSC markers: cells encapsulated in the gel with modulus of $5.3 \mathrm{kPa}$ had the highest growth rate and formed the largest and highest number of tumor spheroids, further exhibiting the highest expression of CSC markers. ${ }^{19}$ Similar to results from other studies discussed earlier, ${ }^{17} \mathrm{CSC}$ marker expression was time-dependent, peaking at 8 days of culture.$^{19}$ In another study, alginate beads with soft $(21 \mathrm{kPa})$, moderate $(70 \mathrm{kPa})$ and hard $(105 \mathrm{kPa})$ stiffness were generated by changing alginate concentration and used to study the effect of 3D matrix stiffness on the biological characteristics of CSCs from human head and neck squamous cell carcinoma (HNSCC). ${ }^{62}$ The authors found that significant CSC enrichment as well as gene expression of stemness markers was achieved in the alginate beads with moderate matrix stiffness. ${ }^{62}$ In the light of these studies, and since stem cells are capable of "remembering" the material stiffness of their environment to determine their differentiation, ${ }^{20}$ it would be prudent to design matrices with appropriate moduli that promote and maintain CSCs.

\section{CONCLUSION}

A summary of the main findings discussed here as well as biomaterials used for the study of CSCs is provided in Table I. Overall, results suggest that growing cancer cells at defined biointerfaces has added benefits over growing them as a monolayer on a tissue culture of polystyrene or even as a nonadherent spheroid culture. Cells grown in biomaterials seem to form spheroids and express CSC characteristics faster, exhibit EMT or MET phenotypes, show higher invasive and tumorigenic potential, and are more resistant to therapies (Table I). Future studies will hopefully bring more insight into the specific biomaterial properties and characteristics that make them applicable to the study of CSCs. Overall, more research is needed to develop and validate suitable and physiologically relevant in vitro models for the expansion of CSCs. We further anticipate that similar strategies can be employed to develop high-throughput CSC therapeutic screening systems to identify novel CSCspecific drugs in the future.

\section{ACKNOWLEDGEMENTS}

This material is based on work supported by the National Science Foundation Grant 1342388 (Y.K.) and by the Stroble Award in Health Sciences by Saint Louis University (S.Z.). Any opinions, findings, and conclusions or recommendations expressed in this material are those of the authors and do not necessarily reflect the views of the National Science Foundation or Saint Louis University, S.D.G.

\section{REFERENCES}

1. T. Reya, S.J. Morrison, M.F. Clarke, and I.L. Weissman, Nature 414, 105 (2001).

2. Y. Kim, K.M. Joo, J. Jin, and D.H. Nam, Int. J. Stem Cells 2, 109 (2009).

3. C.A. O'Brien, A. Kreso, and C.H. Jamieson, Clin. Cancer Res. 16, 3113 (2010). 
4. R. Bjerkvig, B.B. Tysnes, K.S. Aboody, J. Najbauer, and A.J. Terzis, Nat. Rev. Cancer 5, 899 (2005).

5. M. Rahman, L. Deleyrolle, V. Vedam-Mai, H. Azari, M. AbdEl-Barr, and B.A. Reynolds, Neurosurgery 68, 531 (2011).

6. M. Al-Hajj, M.S. Wicha, A. Benito-Hernandez, S.J. Morrison, and M.F. Clarke, Proc. Natl. Acad. Sci. USA 100, 3983 (2003).

7. S.K. Singh, I.D. Clarke, M. Terasaki, V.E. Bonn, C. Hawkins, J. Squire, and P.B. Dirks, Cancer Res. 63, 5821 (2003).

8. C.A. O’Brien, A. Pollett, S. Gallinger, and J.E. Dick, Nature 445, 106 (2007).

9. L. Ricci-Vitiani, D.G. Lombardi, E. Pilozzi, M. Biffoni, M. Todaro, C. Peschle, and R. De Maria, Nature 445, 111 (2007).

10. B.G. Hollier, K. Evans, and S.A. Mani, J Mammary Gland Biol. Neoplasia 14, 29 (2009).

11. K. Polyak and R.A. Weinberg, Nat. Rev. Cancer 9, 265 (2009).

12. K. Sampieri and R. Fodde, Semin. Cancer Biol. 22, 187 (2012).

13. N.A. Lobo, Y. Shimono, D. Qian, and M.F. Clarke, Annu. Rev. Cell Dev. Biol. 23, 675 (2007).

14. T. Borovski, E.M.F. De Sousa, L. Vermeulen, and J.P. Medema, Cancer Res. 71, 634 (2011).

15. F.M. Kievit, S.J. Florczyk, M.C. Leung, K. Wang, J.D. Wu, J.R. Silber, R.G. Ellenbogen, J.S.H. Lee, and M. Zhang, Biomaterials 35, 9137 (2014).

16. D. Fang, T.K. Nguyen, K. Leishear, R. Finko, A.N. Kulp, S. Hotz, P.A. Van Belle, X. Xu, D.E. Elder, and M. Herlyn, Cancer Res. 65, 9328 (2005).

17. W. Rao, S. Zhao, J. Yu, X. Lu, D.L. Zynger, and X. He, Biomaterials 35, 7762 (2014).

18. M.P. Lutolf, P.M. Gilbert, and H.M. Blau, Nature 462, 433 (2009).

19. X. Yang, S.K. Sarvestani, S. Moeinzadeh, X. He, and E. Jabbari, Tissue Eng. Part A 19, 669 (2013).

20. C. Yang, M.W. Tibbitt, L. Basta, and K.S. Anseth, Nat. Mater. 13, 645 (2014).

21. E. Jabbari, S.K. Sarvestani, L. Daneshian, and S. Moeinzadeh, PLoS One 10, e0132377 (2015).

22. M. Baiocchi, M. Biffoni, L. Ricci-Vitiani, E. Pilozzi, and R. De Maria, Curr. Opin. Pharmacol. 10, 380 (2010).

23. S.A. Williams, W.C. Anderson, M.T. Santaguida, and S.J. Dylla, Lab. Invest. 93, 970 (2013).

24. H. Nakshatri, E.F. Srour, and S. Badve, Curr. Stem Cell Res. Ther. 4, 50 (2009).

25. D. Boivin, D. Labbe, N. Fontaine, S. Lamy, E. Beaulieu, D. Gingras, and R. Beliveau, Biochemistry 48, 3998 (2009).

26. E. Binda, A. Visioli, B. Reynolds, and A.L. Vescovi, Front Biosci (Schol Ed) 4, 1235 (2012).

27. J. Lee, S. Kotliarova, Y. Kotliarov, A. Li, Q. Su, N.M. Donin, S. Pastorino, B.W. Purow, N. Christopher, W. Zhang, J.K. Park, and H.A. Fine, Cancer Cell 9, 391 (2006).

28. J.P. Mather, Stem Cells 30, 95 (2012).

29. D. Hanahan and R.A. Weinberg, Cell 144, 646 (2011).

30. A. Rapisarda and G. Melillo, Adv. Cancer Res. 114, 237 (2012).

31. P. Saharinen, L. Eklund, K. Pulkki, P. Bono, and K. Alitalo, Trends Mol. Med. 17, 347 (2011).

32. A.B. Hjelmeland, Q. Wu, J.M. Heddleston, G.S. Choudhary, J. MacSwords, J.D. Lathia, R. McLendon, D. Lindner, A. Sloan, and J.N. Rich, Cell Death Differ. 18, 829 (2011).

33. R.G. Morgan, E. Molnar, R.F. Jones, T.J. Collard, J.D. Lane, A. Greenhough, C. Paraskeva, and A.C. Williams, Br. J. Cancer 112, 714 (2015).
34. E. Lee, J. Yang, M. Ku, N.H. Kim, Y. Park, C.B. Park, J.S. Suh, E.S. Park, J.I. Yook, G.B. Mills, Y.M. Huh, and J.H. Cheong, Cell Death Dis. 6, e1805 (2015).

35. J. Pasquier, N. Abu-Kaoud, H. Abdesselem, A. Madani, J. Hoarau-Vechot, H.A. Thawadi, F. Vidal, B. Couderc, G. Favre, and A. Rafii, BMC Cancer 15, 569 (2015).

36. B. Mognetti, G. La Montagna, M.G. Perrelli, P. Pagliaro, and C. Penna, J. Cell Mol. Med. 17, 287 (2013).

37. L. Li, J. Cole, and D.A. Margolin, Ochsner J. 13, 109 (2013).

38. R. Wurth, A. Bajetto, J.K. Harrison, F. Barbieri, and T. Florio, Front. Cell Neurosci. 8, 144 (2014).

39. A.M. Bleau, A. Agliano, L. Larzabal, A.L. de Aberasturi, and A. Calvo, Histol. Histopathol. 29, 1499 (2014).

40. Y. Kang, Methods Mol. Biol. 568, 7 (2009).

41. S.A. Mani, W. Guo, M.J. Liao, E.N. Eaton, A. Ayyanan, A.Y. Zhou, M. Brooks, F. Reinhard, C.C. Zhang, M. Shipitsin, L.L. Campbell, K. Polyak, C. Brisken, J. Yang, and R.A. Weinberg, Cell 133, 704 (2008).

42. S. Liu, Y. Cong, D. Wang, Y. Sun, L. Deng, Y. Liu, R. Martin-Trevino, L. Shang, S.P. McDermott, M.D. Landis, S. Hong, A. Adams, R. D'Angelo, C. Ginestier, E. CharafeJauffret, S.G. Clouthier, D. Birnbaum, S.T. Wong, M. Zhan, J.C. Chang, and M.S. Wicha, Stem Cell Rep. 2, 78 (2014).

43. M. Luo, M. Brooks, and M.S. Wicha, Curr. Pharm. Des. 21, 1301 (2015).

44. C. Peitzsch, I. Kurth, L. Kunz-Schughart, M. Baumann, and A. Dubrovska, Radiother. Oncol. 108, 378 (2013).

45. L. Chen, Z. Xiao, Y. Meng, Y. Zhao, J. Han, G. Su, B. Chen, and J. Dai, Biomaterials 33, 1437 (2012).

46. K. Deligkaris, T.S. Tadele, W. Olthuis, and A. van den Berg, Sens. Actuators B 147, 765 (2010).

47. K.Y. Lee and D.J. Mooney, Chem. Rev. 101, 1869 (2001).

48. X.-X. Xu, C. Liu, Y. Liu, L. Yang, N. Li, X. Guo, G.-W. Sun, and X.-J. Ma, J. Biotechnol. 177, 1 (2014).

49. F.M. Kievit, S.J. Florczyk, M.C. Leung, O. Veiseh, J.O. Park, M.L. Disis, and M. Zhang, Biomaterials 31, 5903 (2010).

50. M. Leung, F. Kievit, S. Florczyk, O. Veiseh, J. Wu, J. Park, and M. Zhang, Pharm. Res. 27, 1939 (2010).

51. M. Zöller, Nat. Rev. Cancer 11, 254 (2011).

52. G. Chow, J. Tauler, and J.L. Mulshine, J. Biomed. Biotechnol. 2010, 485468 (2010).

53. C. Martinez-Ramos and M. Lebourg, J. Biomed. Mater. Res. B Appl. Biomater. 103, 1249 (2015).

54. S.J. Florczyk, K. Wang, S. Jana, D.L. Wood, S.K. Sytsma, J.G. Sham, F.M. Kievit, and M. Zhang, Biomaterials 34, 10143 (2013).

55. Y.-J. Huang and S.-H. Hsu, Biomaterials 35, 10070 (2014).

56. D.J. Choi, S.M. Choi, H.Y. Kang, H.-J. Min, R. Lee, M. Ikram, F. Subhan, S.W. Jin, Y.H. Jeong, J.-Y. Kwak, and S. Yoon, J. Biotechnol. 205, 47 (2015).

57. L.-J. Liu, J. Zhang, Z.-F. Xiao, B. Dai, M.-Y. Sun, L. Chen, and B. Chen, J. Biomed. Mat. Res. Part B 102, 772 (2014).

58. C.S. Ki, T.-Y. Lin, M. Korc, and C.-C. Lin, Biomaterials 35, 9668 (2014).

59. S. Feng, X. Duan, P.-K. Lo, S. Liu, X. Liu, H. Chen, and Q. Wang, Integr. Biol. 5, 768 (2013).

60. D.E. Discher, P. Janmey, and Y.-L. Wang, Science 310, 1139 (2005).

61. A.J. Engler, S. Sen, H.L. Sweeney, and D.E. Discher, Cell 126,677 (2006).

62. C. Liu, Y. Liu, X.-X. Xu, H. Wu, H.-G. Xie, L. Chen, T. Lu, L. Yang, X. Guo, G.-W. Sun, W. Wang, X.-J. Ma, and X. He, Exp. Cell Res. 330, 123 (2015). 